\title{
Framing the nano-biointeractions by proteomics
}

\author{
S. Sabella*a , G. Maiorano ${ }^{\text {a }}$, L. Rizzello, S. Kote ${ }^{a}$, R. Cingolani ${ }^{\text {, }}$ P. P. Pompa ${ }^{a}$ \\ ${ }^{\mathrm{a}}$ Center for Bio-Molecular Nanotechnology, Italian Institute of Technology \\ Via Barsanti, Arnesano, Lecce, ITALY, 73010 \\ ${ }^{\mathrm{b}}$ Italian Institute of Technology, Central Research Laboratories, \\ Via Morego, Genova, ITALY, 16136
}

\begin{abstract}
Knowledge of the molecular mechanisms underlying the interactions between nanomaterials and living systems is fundamental for providing more effective products for nanomedicine and drug delivery. Controlling the response of cells/bacteria (such as activation of inflammatory processes or apoptosis/necrosis in tumor cells or pathogenic bacteria) by tuning specific properties of the nanomaterials is ultimately the challenging goal. Notably, this may also provide crucial information in the assessment of any toxic risks induced by nanoparticles on humans. However, in studying the nano-biointeractions, it is imperative to take into account the dynamic evolutions of nanoparticles in the biological environments (in terms of protein corona formation, size and charge changes) in synergy with the dynamic events occurring in cells, including signal transduction, metabolic processes, homeostasis and membrane trafficking. In this context, we discuss the impact of analytical technologies, especially in the field of proteomics, which can provide major insights into the processes affecting the NPs surface as well as the cells and bacteria functionalities. In particular, we show that a precise control of the chemical-physical characteristics of the interacting nanoparticles or nanostructures may impact the cells by inducing changes in the proteomic profiles with direct consequences on their viability.
\end{abstract}

Keywords: proteomics, nanoparticles, protein corona, 2D-DIGE, bacteria proteome, biomarkers

\section{INTRODUCTION}

Since its definition ${ }^{1}$, proteomics has been recognized as the new post genomic science with great impact in life science, biology and medicine. Although the lack of a stoichiometric correspondence between the encoding gene and its protein product (due to the numerous and, in some cases, still unknown protein post-translational modifications), ideally, proteomics allows to the identification of the entire set of proteins expressed by genes at a precise temporal cell state, revealing precious information on specific biological processes (e.g., disease processes or drug induced effects), molecular mechanisms for genes regulation, proteins activities, protein-protein interactions, and large scale mapping of subcellular and tissue protein distributions ${ }^{2}$. As such, proteomics has been demonstrated to be an effective tool for toxicology, providing insights in the toxic molecular mechanisms induced by drugs or toxicants in cells or specific tissues (toxicoproteomics). ${ }^{3}$ Moreover, by applying one and two dimensional gel electrophoresis techniques (1- and 2DE) coupled to mass spectrometry (MS), significant differences between control and treated samples (e.g., differential display proteomics) can be quantified, giving information on the different protein expression profiles of cancer cells with respect to normal cells, and on the molecular mechanisms inducing cancerogenicity at protein levels. ${ }^{4}$ Finally, whole protein mapping and biodistribution in diseased or normal tissues as well as protein biomarkers discovery may be also explored by using highly advanced proteomic tools such as LC-MS/MS and quantification methods such as iTRAQ ${ }^{\mathrm{TM}}$, SILAC and others ${ }^{2}$. From a technological point of view, a plethora of technologies and analytical instrumentations, in the field of electrophoresis, mass spectrometry and sensors (e.g., 2-PAGE, 2-DIGE, MALDI-TOF, LC-Q-TOF, SELDI, antibody microarray, etc.) have been developed and improved to address proteomics. Figure 1 reports just some examples of possible proteomic pipelines. Generally, to be successful, such techniques must fit, at least, two principal conditions: separation of complex protein mixtures (for example, whole cellular or tissue lysates, specific cellular components, etc.) and quantification and identification, with the help of bioinformatics tools, of the protein of interest, along with the related biological functionality (if known) associated to its gene.

*stefania.sabella@iit.it; phone 00393396023576; fax 00390832295720; cbn.iit.it

Colloidal Nanocrystals for Biomedical Applications VII, edited by Wolfgang J. Parak, Kenji Yamamoto, Marek Osinski, Proc. of SPIE Vol. 8232, 82320S · (C) 2012 SPIE · CCC code: 1605-7422/12/\$18 · doi: 10.1117/12.910599 
However, all the techniques mentioned in Figure 1 present advantages and limits. In our study, we mainly focused on the use of 1- and 2-DE techniques coupled to MS analyses. Whereas in the case of 1-DE, proteins are separated on the basis of their molecular masses, in the second approach, proteins are discriminated according to their isoelectric point and molecular size. Traditionally, the identification of statistically significant differences between two or more proteomes by 2D-PAGE (Polyacrylamide gel electrophoresis) requires the running and analyses of many gels. In any case, the reproducibility of this method is often hindered by technical variations in sample preparation and gels running conditions that render difficult an appropriate proteins matching between the patterns of treated and control samples (with a consequent difficult quantification of the identified "up" and "down" regulated proteins). 2D-Differential-In-GelElectrophoresis (2D-DIGE) is an effective approach to reduce gel-to-gel variation, by allowing multiplex analyses (by staining proteomes with different dyes) (Figure 1) of two distinct proteomes onto the same gel. Protein matching and the relative comparison are made against a pooled internal standard sample that is used, in all the gels, as an anchor to achieve a better normalization among the gels. ${ }^{5}$

Toxicoproteomics applied to nanotechnology is now emerging as an attractive tool to address the still unanswered questions dealing with nano-biointeractions and NPs-induced toxicity in living systems. For example, it has been reported that NPs suspended in biological fluids adsorb a set of different proteins (referred to as protein corona) ${ }^{6-7}$ whose composition and affinity kinetics strongly depend on particles surface, size, and concentration. Hence, protein coating, surrounding and hiding NPs surface, plays a pivotal role in determining the effective size, surface charge, aggregation state of the newly formed hybrid bio-nanomaterials, influencing biodistribution and, finally, triggering the beneficial or hazardous biological effect. ${ }^{8-9}$ The exposed protein corona may, in turn, activate specific epitopes on the cells surface, thus allowing the recognition and the activation of specific receptors and cellular functions. ${ }^{10}$ Furthermore, it has been shown that poly (acrylic acid)-conjugated gold nanoparticles, selectively binding fibrinogen contained in the plasma, induce its unfolding and the subsequent specific activation of Mac-1 integrin receptor. This activation primes the NF-kB signaling pathway, leading to the release of inflammatory factors. ${ }^{11}$ Finally, by applying "omic" techniques and in particular 2D-DIGE, it has been recently demonstrated that AuNPs induce toxicity by a direct damage of the cellular endoplasmic reticulum (ER). ${ }^{12}$

In this scenario, we describe herein the application of different proteomic approaches to characterize the protein corona involved in the interaction of AuNPs with different cellular media and to frame the proteomic response of $E$. Coli bacteria upon interaction with nanostructured gold substrate. In particular, we show the application of different proteomic workflows. In the first case, we applied the 1-DE/MS analyses to characterize the temporal evolution of protein coronas of differently sized gold NPs and their modulating effects in inducing cellular toxicity. Importantly, in the second approach, by 2D-DIGE and LC-MS/MS, we demonstrate how nanoscale changes in the nanoroughness of a gold substrates may impact on the bacteria functionality by triggering changes at post-translational levels, in the bacteria proteome. Finally, many proteins involved in the molecular mechanisms leading to the loss of bacteria fimbrial structures are highlighted.

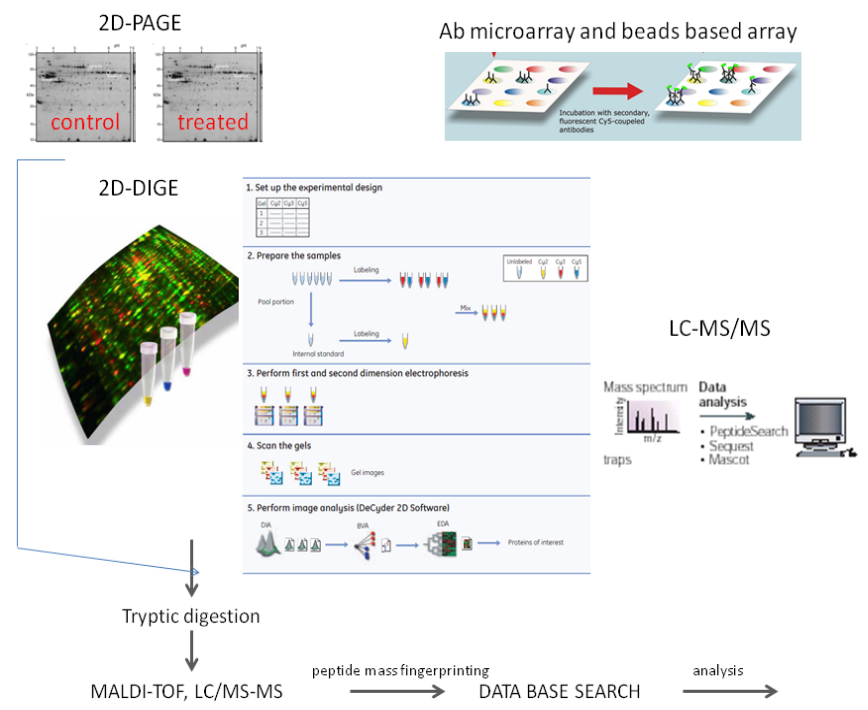

Figure 1 Examples of analytical platforms for proteomics studies 


\section{RESULT AND DISCUSSIONS}

\subsection{Characterization of the time-dependent evolution of hard protein corona of AuNPs by proteomics}

Many methods have been applied to investigate protein corona ex situ, all showing advantages and limits. Among them, centrifugation method has been recently recognized to provide useful information on what is usually called "hard protein corona". In general, once the NPs are incubated with serum proteins, the resulting protein pellet is isolated by centrifugation and accurate washing. Afterwards, the stable protein/NP complexes are analyzed by following the proteomic workflow reported in Figure 2 that explores the use of SDS-PAGE for proteins separation and MS analyses for protein identification. In particular, in our study, we aimed at the characterization of hard protein corona obtained from differently sized AuNPs $(15,40$ and $80 \mathrm{~nm}$ ) in the presence of two cell culture media (DMEM, RPMI) enriched with FBS, as proteins source. Furthermore, a semi-quantitative description of the temporal evolution of protein coronas out of cellular medium solution (ex situ) was performed by applying a semi-quantitative approach based on densitometry analysis (Figure 2). Hence, after centrifugation, we collected pellets at two specific temporal points (namely, $1 \mathrm{~h}$ and 48 h) that, on the basis of previously reported spectroscopic measurements of protein coronas in solution, ${ }^{13}$ are known to correspond to the starting and plateau phases of protein corona formation onto the NPs surfaces. Such procedure likely reproduces an off-line snapshot of the kinetic of NPs corona in solution. Importantly, in order to compare the different protein electrophoretic lines, we incubated batches of AuNPs at concentrations giving constant values of the total surface area of the NPs for all the sizes. Representative gels (Figure 2A) showed rich protein profiles for all the AuNPs sizes in both the cell culture media. Densitometric analyses indicated that, whereas at $1 \mathrm{~h}$ of incubation the total amount of adsorbed proteins roughly increases as a function of the nanoparticle sizes, with no particular differences between the two media, after $48 \mathrm{~h}$ (plateau phase), DMEM incubated AuNPs produced a higher total amount of hard protein coronas (as also previously evidenced by TEM images). ${ }^{13}$ These data are in good agreement with our previous results obtained by means of in situ characterization (i.e., in medium solutions), ${ }^{13}$ supporting the recent idea that inspection of protein corona by separation methods may be useful to study the complex dynamics underlying proteins/NP interactions in solution. ${ }^{14}$ We analyzed the relative distribution of protein classes as a function of NPs size and cellular media. Interestingly, gel images and densitometric analyses revealed that protein patterns strongly differ with respect to the relative distribution of serum proteins (data not reported). In particular, bands belonging to 1-9 classes (280-100 KDa) were grouped in the high molecular weight proteins (H.M.W.), 10-21 classes (100-35 kDa) in the medium molecular weight proteins (M.M.W.), bands 22-31 (35-10 kDa) in the low molecular weight (L.M.W.) range. On the basis of their electrophoretic mobility and mass, we can argue that most represented proteins of FBS, such as BSA and Fibrinogen, are in 12-15 classes range. We can observe that such species are not predominantly represented in the gel lines of protein coronas. This suggest that, in the serum mixture, complex mechanisms of competition among highly affine protein molecules lead NPs to act as concentrators of less abundant serum proteins, while BSA and Fibrinogen only partially interact with the NPs surface (Figure 2) ${ }^{7,15-19}$ Such an effect was found to be similar in both the cellular media, with a slight dependence on the NPs size. Moreover, histograms relative to the band intensity of the three grouped classes (i.e., H.M.W, M.M.W and L.M.W) revealed further differences in the kinetics of proteins distribution in the hard protein corona (Figure 2B). In particular, at 1 h, H.M.W. and L.M.W. proteins are strongly up-represented with respect to fetal bovin serum (control), while M.M.W. proteins are down represented (Figure 2B). After 48 hours, the intensity of M.M.W. protein classes increases at expense of the L.M.W. proteins (Figure 2B), while layers composed by high molecular weight protein do not show particular changes over time. ${ }^{14,18-21}$ This suggests that this latter class is less susceptible than the other groups to the dynamic protein exchange occurring during the formation of protein corona. Since increasing evidences suggest that the specific molecular composition of protein corona may play a fundamental role in triggering biological responses, ${ }^{7,9,15,16}$ the above characterizations are of particular interest.

The gel bands of interest were excised, digested and analyzed by LC-MS/MS. The main identified protein species adsorbed onto the metallic NPs surfaces are reported on the Table in Figure 2. We found out important proteins involved in key biological processes, such as transport and trafficking (Apolipoprotein A1, Transferrin, Vitamin D-binding protein, etc.), blood coagulation (Protein $\mathrm{C}$ inhibitor, antithrombin III, Coagulation factor V, etc.) and tissue development (Fibulin 1, periostin, Thrombospondin-1, Galectin 3 binding protein, etc.). ${ }^{7,18,19,22}$ On the basis of their molecular weight, such proteins are present especially in the M.M.W. and L.M.W. groups. Hence, they may be susceptible to the exchange kinetics evidenced in Figure 2B. Such changes, occurring within the experimental temporal windows typical of cell viability tests, might differently affect the outcomes of nanotoxicity tests, due to the specific biological influence of protein coronas. 

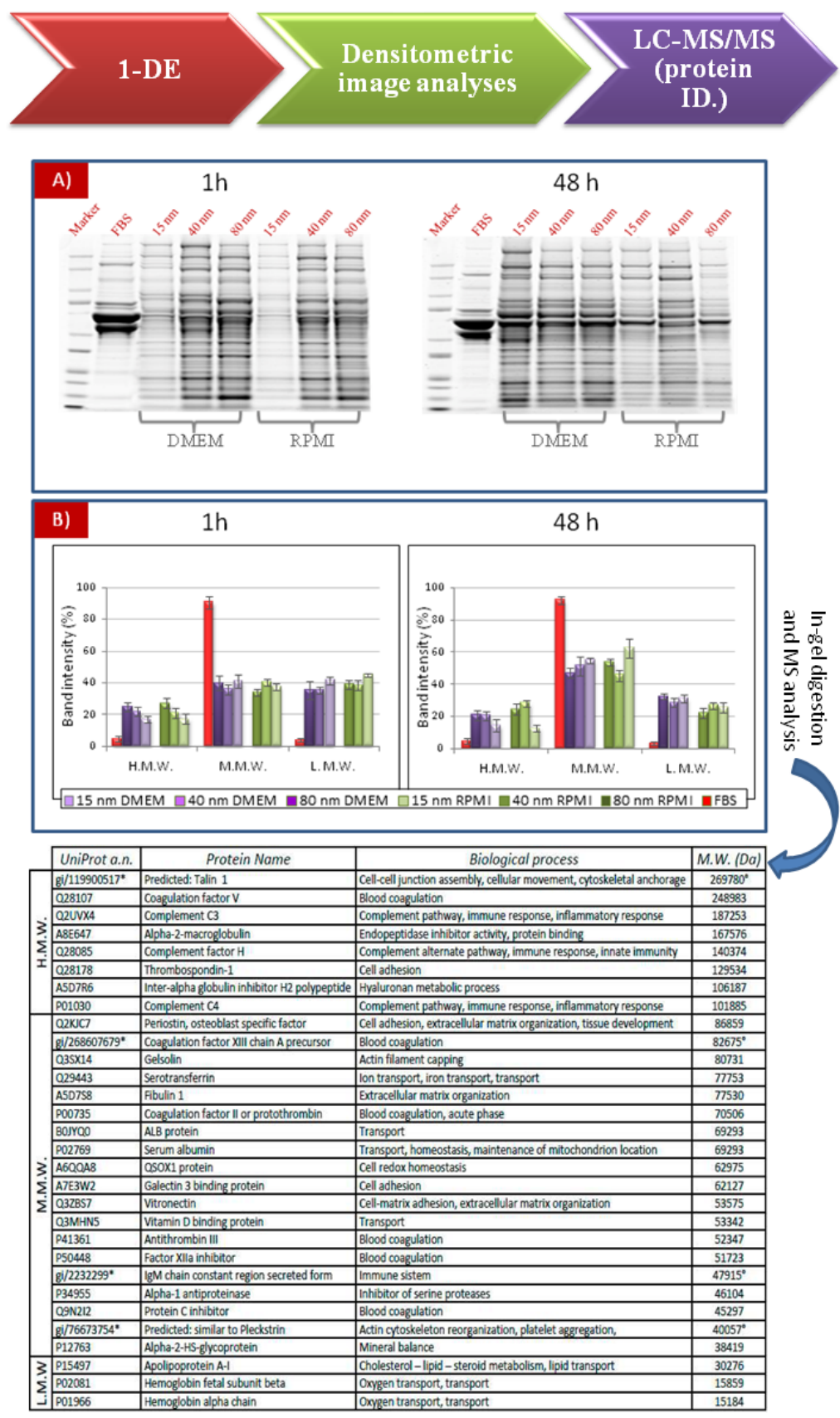

Figure 2: Proteomic workflow for the "ex situ" characterization of the protein corona onto AuNPs in the presence of the cell culture media, enriched with FBS as proteins source. A) Representative SDS-PAGE of protein corona obtained from 15, 40 and $80 \mathrm{~nm}$ AuNPs, after incubation with DMEM and RPMI at $37^{\circ} \mathrm{C}$ for 1 hour and 48 hours. (B) Semiquantitative densitometry analyses of gel bands at two different time points of protein corona formation $(1 \mathrm{~h}$ and $48 \mathrm{~h})$. Approximately 30 gel bands were experimentally detected as single peaks, whose intensity percentages were plotted $v s$ band electrophoretic position (data not reported). Classes 1-31 are ranged starting from high to low molecular weights. Plot of band intensity vs pooled gel bands, which were grouped as reported: 1-9 as H.M.W., 10-20 as M.M.W. and 21-31 as L.M.W., according to the experimental position. Data are reported as mean values of three independent experiments. Identified proteins by LC-MS/MS and their relative biological functions were reported in the Table. 


\subsection{Bacteria proteome is influenced by bacteria/nanorough films interaction}

To characterize the nano-biointeractions we explored another proteomic approach using the combination of 2D-DIGE technique and LC-MS/MS. This proteomic workflow (Figure 3) led us to investigate the entire proteomic profile of $E$. coli, upon interaction with substrates at controlled levels of nanoroughness. 2D-DIGE is a well established and powerful method that allows understanding and clarifying specific metabolic pathways of cells by highlighting up- or downexpression of proteins. ${ }^{23-25}$ Figure 3(top) shows a representative 2D gel (in single channels and overlaid image) in which $\sim 1500$ protein spots of $E$. coli grown on both glass and nanorough gold substrates were identified. We used strips with a 4-7 $\mathrm{pH}$ gradient for the first dimension to better resolve the bacterial proteomic profile. The quality of our 2D-DIGE experiments was demonstrated by a variance below $15 \%$ for 1500 spots within the biological replicates ${ }^{26}$ at the same time, also the Principal Component Analysis (PCA) (Figure 3bottom) clearly displayed two well resolved populations. Yellow spots (which represent the population of control proteins pool) are in fact grouped together in the left side of the Spot Maps and are well separated from the red spots (representing the treated population) ${ }^{27}$ (Figure 3 bottom). Among the $\sim 1500$ protein spots in the 2D gel, we identified 15 of them which were differentially expressed (up- or downregulated) with significant statistical validity [t-test $<0.02$ between glass (control) and nanorough (treated)] (no significant differences were detected between glass and flat gold surface). These 15 significant spots, representative of 15 proteins, showed up and down trends of regulation, as also quantified by the Extended Data Analysis (EDA) (data not reported). Protein identification was performed by picking up the 15 significant spots, from the corresponding preparative gel, and carrying out LC-MS/MS mass spectrometry analyses. We observed that the presence of nanotopography alone, without chemical or biological external stimuli/surface modifications, induces a different expression of proteins involved in transcription regulation, transport of oligopeptides and aminoacids, energy metabolism, stress response, and synthesis and modification of macromolecules (Table II). In particular, we found that some proteins involved in the general stress response were regulated in the nanorough samples. For instance, the DNA protection during starvation protein (Dps), that represents one example of defense against oxidative DNA damage in actively growing cells ${ }^{28}$ was up-regulated. At the same time, also the OsmC stress-inducible membrane protein (whose transcription is typically induced at elevated osmotic pressure) codified by the osmC gene ${ }^{29}$ was up-regulated. Such latter finding is also confirmed by the down-regulation of the H-NS transcriptional regulator, which is a histone-like protein able to repress the expression of numerous genes, including osmC. ${ }^{30}$ We also found that several proteins involved in general cell processes were regulated; in this frame, the over-expression of the YaeT complex is of particular interest, as it is required for the Outer Membrane $\beta$-barrel Proteins (OMPs) assembly, that is important for a proper maintenance of the outer membrane, required for cell viability. ${ }^{31}$ In addition, the NusA protein, involved in transcription termination in the tryptophan operon of E. coli, ${ }^{32}$ and the Periplasmatic Histidine Binding Protein (HBP), that is an initial receptor in the process of active transport across cell membranes and/or chemotaxis, ${ }^{31}$ are both up-regulated in the bacteria interacting with the nanorough surfaces. On the other side, the Oligopeptide transport OppA, ${ }^{33}$ the Outer Membrane Protein A (OMPA), that plays a structural role in the integrity of the bacterial cell surface, ${ }^{34}$ and Protein YgiW are downexpressed. These observations indicate that nanostructured surfaces strongly affect $E$. coli cells, leading to general stress processes in bacteria, which activate defense mechanisms against DNA or membrane damage. Moreover, we detected proteins important for the synthesis and modification of macromolecules. The $\beta$-subunit of the Glycyl-tRNA synthetase, an important enzyme in protein synthesis, ${ }^{35}$ and the Dihydrodipicolinate synthase, a crucial target enzyme of many antibiotics ${ }^{36}$ resulted over-expressed. On the other hand, the Uracil phosphoribosyltransferase, which is the precursor for all pyrimidine nucleotides (pyrimidine salvage enzyme), ${ }^{37}$ is down-regulated. Finally, we found that the energy metabolism proteins Transketolase $2^{38}$ and Pyruvate kinase ${ }^{39}$ were both up-regulated in treated samples. Overall, such protein pattern suggests that $E$. coli undergoes important changes in the metabolic pathways upon interaction with nanostructured surfaces, confirming that nanoroughness alone causes a general stress condition in the bacteria. A possible explanation is that the nanotopography causes a significantly reduced contact area of the bacteria with the underlying substrate, likely perturbing their anchoring mechanisms. This might also lead to damages of the outer membrane of adherent $E$. coli, which consequently rearrange its protein expression profile up-regulating enzymes involved in the protection of DNA, aminoacid synthesis, energy production, regulation and rearrangement of the external membrane; at the same time, E. coli down-regulates some transport proteins and enzymes related to DNA synthesis, possibly trying to avoid mistakes and/or damages during base synthesis. 

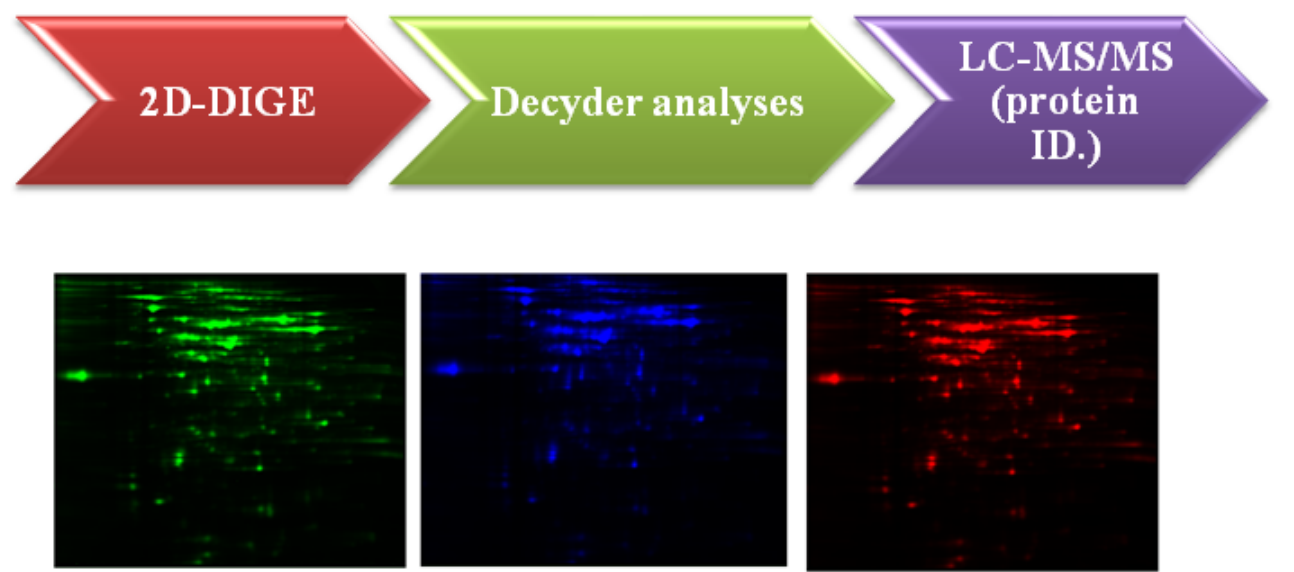

Test Cy3
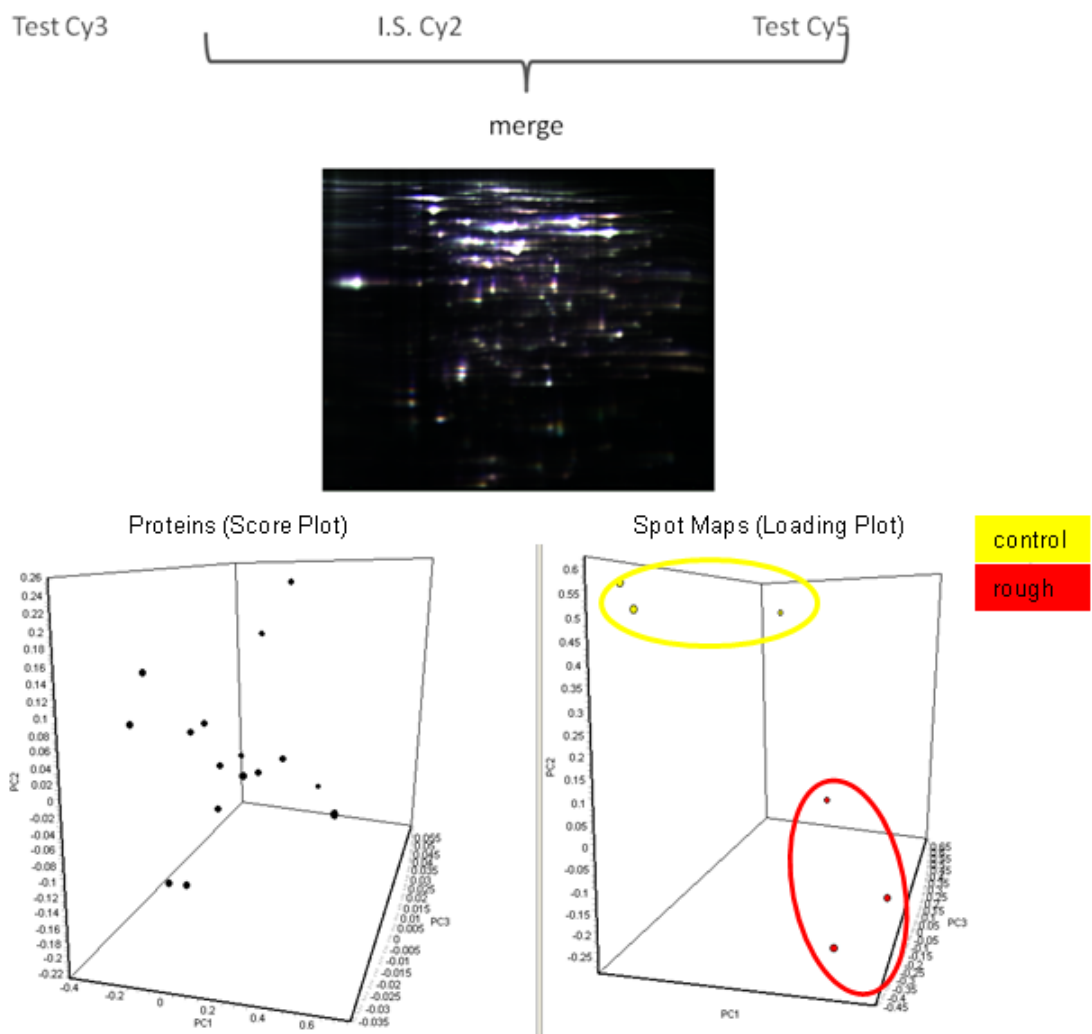

Figure 3 2-D DIGE and Principal Component Analysis (PCA) of bacteria extracts upon treatment with control and nanorough gold substrates. Top): overlay of whole proteome of bacteria treated onto flat (Cy3) and rough gold substrate (Cy5), and pooled internal standard (Cy2). The three samples show overlap based on distinct color of protein spots; bottom) Clustering analysis of proteins by PCA. A filter including all proteins that were present in $>75 \%$ spot maps was applied. Previously, a subset considering those proteins whose expression varied within the 95th confidence level (Student's t test; $\mathrm{p}<0.02$ ) was created. 


\begin{tabular}{|c|c|c|c|c|c|c|c|}
\hline $\begin{array}{c}\text { Master } \\
\text { spot N. }\end{array}$ & Protein ID & Protein name & Av. ratio & t test $t^{\sigma}$ & Score & Coverage & $\begin{array}{c}\text { Theoretical } \\
\text { mass }(K D a)^{c} ; p f^{\epsilon}\end{array}$ \\
\hline
\end{tabular}

Cell Processes

\begin{tabular}{|c|c|c|c|c|c|c|c|}
\hline 11 & gi/15799859 & $\begin{array}{l}\text { Outer membrane protein } \\
\text { assembly factor yaeT }\end{array}$ & +1.19 & 0.0071 & 1668 & 45 & $90.6 ; 4.93$ \\
\hline 43 & gi/15803711 & $\begin{array}{c}\text { Transcription elongation } \\
\text { factor NusA }\end{array}$ & +1.19 & 0.0083 & 1159 & 56 & $55 ; 4.53$ \\
\hline 157 & $\mathrm{gi} / 170080870$ & $\begin{array}{l}\text { Oligopeptide transport } \\
\text { periplasmatic subunit }\end{array}$ & -1.34 & 0.0094 & 863 & 52 & $61 ; 6.05$ \\
\hline 155 & $\mathrm{gi} / 15801469$ & Oligopeptide transport & -1.32 & 0.0009 & 638 & 51 & $61 ; 5.95$ \\
\hline 658 & gi/194436225 & $\begin{array}{l}\text { Periplasmatic histidine } \\
\text { binding protein (HBP) }\end{array}$ & +1.23 & 0.0067 & 859 & 62 & $28.5 ; 5.30$ \\
\hline 1080 & $\mathrm{gi} / 15800816$ & Outer membrane protein $\mathrm{A}$ & -1.38 & 0.0148 & 309 & 26 & $37.2 ; 5.99$ \\
\hline 1235 & gi/15803566 & Hypothetical protein Z4376 & -1.45 & 0.0045 & 466 & 49 & $14 ; 5.08$ \\
\hline
\end{tabular}

Synthesis and modification of macromolecules

\begin{tabular}{|c|c|c|c|c|c|c|c|}
\hline 47 & gi/16131430 & Glycyl-tRNA synthetase & +1.3 & 0.0079 & 1111 & 47 & $76.9 ; 5.29$ \\
\hline 482 & $\mathrm{gi} / 15803001$ & Dihydrodipicolinate synthase & +1.07 & 0.0058 & 508 & 40 & $31.6 ; 5.98$ \\
\hline 913 & $\mathrm{gi} / 38704095$ & $\begin{array}{c}\text { Uracil } \\
\text { phosphoribosyltransferase }\end{array}$ & -1.17 & 0.0024 & 650 & 78 & $22.5 ; 5.32$ \\
\hline
\end{tabular}

Energy metabolism

\begin{tabular}{|c|c|c|c|c|c|c|c|}
\hline 56 & gi/16130390 & Transketolase 2 & +1.24 & 0.014 & 763 & 30 & $73.2 ; 5.86$ \\
\hline 220 & $\mathrm{gi} / 15802267$ & Pyruvate kinase & +1.3 & 0.0074 & 99 & 13 & $51.5 ; 6.44$ \\
\hline
\end{tabular}

General stress response

\begin{tabular}{|c|c|c|c|c|c|c|c|}
\hline 1060 & $\mathrm{gi} / 15800564$ & $\begin{array}{c}\text { DNA protection during } \\
\text { starvation protein }\end{array}$ & +1.82 & 0.0146 & 585 & 73 & $18.6 ; 5.72$ \\
\hline 1127 & $\mathrm{gi} / 15801465$ & DNA-binding protein H-NS & -1.05 & 0.0049 & 448 & 54 & $15.5 ; 5.43$ \\
\hline 1220 & $\mathrm{gi} / 15801655$ & $\begin{array}{c}\text { Osmotically inducible, stress- } \\
\text { inducible membrane protein }\end{array}$ & +1.52 & 0.0057 & 96 & 20 & $15.2 ; 5.57$ \\
\hline
\end{tabular}

Table II. Summary of protein identification based on peptide mass fingerprinting/peptide sequencing and database searching approaches using LC-MS/MS analyses. The protein are classified according to their biological function, with the average ratio and statistical data (obtained by DeCyder software), and the data from Mascot identifications.

${ }^{a}$ Average volume ratio (treated/control) and t test $\mathrm{p}$ value, quantified by DeCyder BVA module. The average ratio value indicates the standardized volume ratio between the two groups (indicated as $\log$ of the true ratio measurements). The values are displayed in the range from $-\infty$ to -1 for decreased expression and from +1 to $+\infty$ for increased expression.

${ }^{\mathrm{b}}$ Score: Mascot search algorithm reports ion scores as $-10^{*} \log (\mathrm{P})$, where $\mathrm{P}$ is the probability that the observed match is a random event. Scores $>38$ indicate identity or extensive homology $(p<0.05)$.

${ }^{\mathrm{c}}$ Calculated automatically by Mascot. 


\section{REFERENCES}

[1] Wilkins M.R., Sanchez J.C., Gooley A.A., Appel R.D., Humphery-Smith I., Hochstrasser D.F., and Williams K.L., "Progress with proteome projects: why all proteins expressed by a genome should be identified and how to do it," Biotechnology \& genetic engineering reviews, 13, 19-50 (1996).

[2] Ong S.-E., and Mann M., "Mass spectrometry-based proteomics turns quantitative," Nat Chem Biol, 1(5), 252$262(2005)$.

[3] Merrick B.A., and Witzmann F.A., "The role of toxicoproteomics in assessing organ specific toxicity" EXS, 99, 367-400 (2009).

[4] Nomura D.K., Dix M.M., and Cravatt B.F., "Activity-based protein profiling for biochemical pathway discovery in cancer" 10, 631-638 (2010).

[5] Goldfarb M., "Computer analysis of two-dimensional gels," J Biomol Tech, 18(3), 143-6 (2007).

[6] Cedervall T., Lynch I., Lindman S., Berggård T., Thulin E., Nilsson H., Dawson K.A., and Linse S., "Understanding the nanoparticle-protein corona using methods to quantify exchange rates and affinities of proteins for nanoparticles," Proceedings of the National Academy of Sciences, 104(7), 2050-2055 (2007).

[7] Lundqvist M., Stigler J., Elia G., Lynch I., Cedervall T., and Dawson K.A., "Nanoparticle size and surface properties determine the protein corona with possible implications for biological impacts," Proceedings of the National Academy of Sciences, 105(38), 14265-14270 (2008).

[8] Nel A.E., Mädler L., Velegol D., Xia T., Hoek E.M., Somasundaran P., Klaessig F., Castranova V., and Thompson M., "Understanding biophysicochemical interactions at the nano-bio interface," Nat Mater, 8(7), 543-557 (2009).

[9] Dutta D., Sundaram S.K., Teeguarden J.G., Riley B.J., Fifield L.S., Jacobs J.M., Addleman S.R., Kaysen G.A., Moudgil B.M., and Weber T.J., "Adsorbed Proteins Influence the Biological Activity and Molecular Targeting of Nanomaterials," Toxicological Sciences, 100(1), 303-315 (2007).

[10] Lynch I., Dawson K.A., and Linse S., "Detecting Cryptic Epitopes Created by Nanoparticles," Sci. STKE, 2006(327), pe14- (2006).

[11] Deng Z.J., Liang M., Monteiro M., Toth I., and Minchin R.F., "Nanoparticle-induced unfolding of fibrinogen promotes Mac-1 receptor activation and inflammation" Nat Nano, 6(1), 39-44 (2011).

[12] Tsai Y.Y., Huang Y.H., Chao Y.L., Hu K.Y., Chin L.T., Chou S.H., Hour A.L., Yao Y.D., Tu C.S., Liang Y.J., Tsai C.Y., Wu H.Y., Tan S.V., and Chen H.M., "Identification of the Nanogold Particle-Induced Endoplasmic Reticulum Stress by Omic Techniques and Systems Biology Analysis," ACSNano (2011, in press).

[13] Maiorano G., Sabella S., Sorce B., Brunetti V., Malvindi M.A., Cingolani R., and Pompa P.P., "Effects of Cell Culture Media on the Dynamic Formation of Protein-Nanoparticle Complexes and Influence on the Cellular Response," ACS Nano, 4(12), 7481-7491 (2010).

[14] Walczyk D., Bombelli F.B., Monopoli M.P., Lynch I., and Dawson K.A., "What the Cell "Sees" in Bionanoscience," Journal of the American Chemical Society, 132(16), 5761-5768 (2010).

[15] Aggarwal P., Hall J.B., McLeland C.B., Dobrovolskaia M.A., and McNeil S.E., "Nanoparticle interaction with plasma proteins as it relates to particle biodistribution, biocompatibility and therapeutic efficacy," Advanced Drug Delivery Reviews, 61(6), 428-437 (2009).

[16] Dobrovolskaia M.A., Patri A.K., Zheng J., Clogston J.D., Ayub N., Aggarwal P., Neun B.W., Hall J.B., and McNeil S.E., "Interaction of colloidal gold nanoparticles with human blood: effects on particle size and analysis of plasma protein binding profiles," Nanomedicine, 5(2), 106-117 (2009).

[17] Vroman L., and Adams A.L., "Adsorption of Proteins out of Plasma and Solutions in Narrow Spaces," Journal of Colloid and Interface Science, 111(2), 391-402 (1986).

[18] Cedervall T., Lynch I., Foy M., Berggård T., Donnelly S.C., Cagney G., Linse S., and Dawson K.A., "Detailed Identification of Plasma Proteins Adsorbed on Copolymer Nanoparticles," Angewandte Chemie International Edition, 46(30), 5754-5756 (2007).

[19] Lynch I., and Dawson K.A., "Protein-nanoparticle interactions," Nano Today, 3(1-2), $40-47$ (2008).

[20] Lynch I., Cedervall T., Lundqvist M., Cabaleiro-Lago C., Linse S., and Dawson K.A., "The nanoparticleprotein complex as a biological entity; a complex fluids and surface science challenge for the 21st century," Advances in Colloid and Interface Science, 134-135(0), 167-174 (2007).

[21] Casals E., Pfaller T., Duschl A., Oostingh G.J., and Puntes V., "Time Evolution of the Nanoparticle Protein Corona," ACS Nano, 4(7), 3623-3632 (2010). 
[22] Alberts A., Johnson A., Lewis J., Raff M., Roberts K., and Walter P., "Molecular Biology of the Cell”; Garland Science: New York, 2002.

[23] Di Michele M., Della Corte A., Cicchillitti L., Del Boccio P., Urbani A., Ferlini C., Scambia G., Donati M.B., and Rotilio D., "A proteomic approach to paclitaxel chemoresistance in ovarian cancer cell lines," Biochimica et Biophysica Acta (BBA) - Proteins \&amp; Proteomics, 1794(2), 225-236 (2009).

[24] Tumani H., Lehmensiek V., Lehnert S., Otto M., and Brettschneider J., "2D DIGE of the cerebrospinal fluid proteome in neurological diseases," Expert Review of Proteomics, 7(1), 29-38 (2010).

[25] Yang X., Liu J., He H., Zhou L., Gong C., Wang X., Yang L., Yuan J., Huang H., He L., Zhang B., and Zhuang $\mathrm{Z}$., " $\mathrm{SiO}_{2}$ nanoparticles induce cytotoxicity and protein expression alteration in $\mathrm{HaCaT}$ cells," Particle and Fibre Toxicology, 7(1), 1 (2010).

[26] Stephens A.N., Pereira-Fantini P.M., Wilson G., Taylor R.G., Rainczuk A., Meehan K.L., Sourial M., Fuller P.J., Stanton P.G., Robertson D.M., and Bines J.E., "Proteomic Analysis of the Intestinal Adaptation Response Reveals Altered Expression of Fatty Acid Binding Proteins Following Massive Small Bowel Resection," Journal of Proteome Research, 9(3), 1437-1449 (2009).

[27] Rodríguez-Piñeiro A.M., Blanco-Prieto S., Sánchez-Otero N., Rodríguez-Berrocal F.J., de la Cadena M.P., "On the identification of biomarkers for non-small cell lung cancer in serum and pleural effusion," Journal of Proteomics, 73(8), 1511-1522 (2010).

[28] Martinez A., and Kolter R., "Protection of DNA during oxidative stress by the nonspecific DNA-binding protein Dps," Journal of Bacteriology, 179(16), 5188-94 (1997).

[29] Davalos-Garcia M., Conter A., Toesca I., Gutierrez C., and Cam K., "Regulation of osmC Gene Expression by the Two-Component System rcsB-rcsC in Escherichia coli," Journal of Bacteriology, 183(20), 5870-5876 (2001).

[30] Bouvier J., Gordia S., Kampmann G., Lange R., Hengge-Aronis R., and Gutierrez C., "Interplay between global regulators of Escherichia coli: effect of RpoS, Lrp and H-NS on transcription of the gene osmC," Molecular Microbiology, 28(5), 971-980 (1998).

[31] Wu T., Malinverni J., Ruiz N., Kim S., Silhavy T.J., and Kahne D., "Identification of a Multicomponent Complex Required for Outer Membrane Biogenesis in Escherichia coli," Cell, 121(2), 235-245 (2005).

[32] Farnham P.J., Greenblatt J., and Platt T., "Effects of NusA protein on transcription termination in the tryptophan operon of Escherichia coli," Cell, 29(3), 945-951 (1982).

[33] Goodell E.W., and Higgins C.F., "Uptake of cell wall peptides by Salmonella typhimurium and Escherichia coli," Journal of Bacteriology, 169(8), 3861-3865 (1987).

[34] Koebnik R., Locher K.P., and Van Gelder P., "Structure and function of bacterial outer membrane proteins: barrels in a nutshell," Molecular Microbiology, 37(2), 239-253 (2000).

[35] Schimmel P.R., and Soll D., "Aminoacyl-tRNA synthetases: general features and recognition of transfer RNAs," Annu Rev Biochem, 48, 601-48 (1979).

[36] Dobson R.C., Griffin M.D., Roberts S.J., and Gerrard J.A., "Dihydrodipicolinate synthase (DHDPS) from Escherichia coli displays partial mixed inhibition with respect to its first substrate, pyruvate," Biochimie, 86(45), 311-315.

[37] Andersen P.S., Smith J.M., and Mygind B., "Characterization of the upp gene encoding uracil phosphoribosyltransferase of Escherichia coli K12,” European Journal of Biochemistry, 204(1), 51-56 (1992).

[38] Iida A., Teshiba S., and Mizobuchi K., "Identification and characterization of the tktB gene encoding a second transketolase in Escherichia coli K-12," Journal of Bacteriology, 175(17), 5375-5383 (1993).

[39] Mattevi A., Valentini G., Rizzi M., Speranza M.L., Bolognesi M., and Coda A., "Crystal structure of Escherichia coli pyruvate kinase type I: molecular basis of the allosteric transition," Structure (London, England : 1993), 3(7), 729-741 (1995). 